\section{MINIATURE AXIAL FLOW PUMP FOR VENTRICULAR ASSISTANCE IN CHILDREN AND SMALL ADULTS}

We investigated the efficacy of the Jarvik 2000 intraventricular assist device (Jarvik Research, Inc., New York, N.Y.) in an ovine model. The device is an axial flow pump measuring $1.8 \mathrm{~cm}$ in diameter by $5 \mathrm{~cm}$ long, has a displacement volume of $12 \mathrm{ml}$, and can deliver flow from 2 to $7 \mathrm{~L} / \mathrm{min}$. Seven devices were implanted through a left thoracotomy into the left ventricle with an outflow graft to the descending aorta. Animals were treated with warfarin sodium and aspirin to maintain prothrombin times approximately 1.5 times control. Animals were followed up for 3 to 123 days. Two animals died of operative complications at days 3 and 5 . One device failed at 58 days because of thrombus formation at the inflow side of the impeller. The remaining four animals were killed at days $19,42,42$, and 123 , respectively, because of broken electric power cables. Hematocrit values rose significantly higher than preoperative levels $(22.8 \% \pm 3.8 \%$ to $30.5 \% \pm 3.4 \%)$; premortem elevations of values higher than baseline values of plasma free hemoglobin $(10.4 \pm 7.8 \mathrm{mg} / \mathrm{dl}$ to $17.1 \pm 7.4 \mathrm{mg} / \mathrm{dl})$ and lactate dehydrogenase $(391.5 \pm 113.7$ units $/ L$ to $771.2 \pm 370.8$ units $/ L)$ were statistically insignificant. Serum creatinine and bilirubin levels were normal. No end-organ dysfunction arising from long-term support was evident clinically or at postmortem examination, nor was there any evidence of embolism or damage to intracardiac structures. We found the Jarvik 2000 intraventricular assist device to be easily implantable, safe, nonhemolytic, and able to provide physiologic flow with power requirements under 10 watts. (J ThoraC Cardiovasc Surg 1996;111:13-8)

Richard J. Kaplon, MD, ${ }^{a}$ Mehmet C. Oz, MD, ${ }^{\text {a }}$ Pawel A. Kwiatkowski, MD, Howard R. Levin, MD, ${ }^{a}$ Aamir S. Shah, MD, ${ }^{a}$ Robert K. Jarvik, MD, ${ }^{b}$ and Eric A. Rose, MD, ${ }^{\mathrm{a}}$ New York, N.Y.
W ith approximate 1 - and 5-year survivals of $80 \%$ and $60 \%$, respectively, cardiac transplantation has become accepted as the treatment of choice for patients with end-stage heart failure.' Unfortunately, the critical shortage of available donor organs is the foremost limitation to heart transplantation and is such that the number of patients on the United Network for Organ Sharing (UNOS) waiting list exceeds the number of patients who undergo

From the Cardiopulmonary Transplantation Research Laboratory, Division of Cardiothoracic Surgery, Columbia-Presbyte-

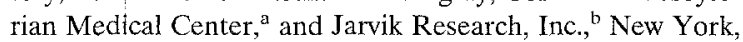
N.Y

Received for publication August 12, 1994.

Accepted for publication March 8, 1995.

Address for reprints: Richard J. Kaplon, MD, Division of Cardiothoracic Surgery, Columbia-Presbyterian Medical Center, 177 Fort Washington Ave., MHB 7-435, New York, NY 10032.

Copyright 1996 by Mosby-Year Book, Inc.

$0022-5223 / 96 \$ 5.00+0 \quad \mathbf{1 2 / 1 / 6 4 5 9 3}$ transplantation annually almost fourfold. ${ }^{2}$ Despite continually expanding donor criteria, many patients who might benefit from cardiac transplantation are considered too old or too sick to be offered this therapeutic modality and as many as $20 \%$ die while awaiting an organ. ${ }^{3,4}$

Since Reemtsma and colleagues ${ }^{5}$ first demonstrated that "bridging" to heart transplantation with mechanical assistance could be successfully done, left ventricular assist devices (LVADs) have become more widely used. ${ }^{6,7}$ Unfortunately, placement of the two devices specifically designed for long-term mechanical circulatory support, the HeartMate 1000 IP device (Thermocardiosystems Inc., Woburn, Mass.) and the Novacor LVAS (Novacor Medical Corp., Oakland, Calif.), has been technically unfeasible in patients with body surface areas less than $1.5 \mathrm{~m}^{2}$, thus excluding small women and children. ${ }^{8}$

In contrast to pusher-plate devices, axial flow pumps are small. Further, such devices are quiet, easily implantable, and relatively inexpensive. ${ }^{9}$ To 


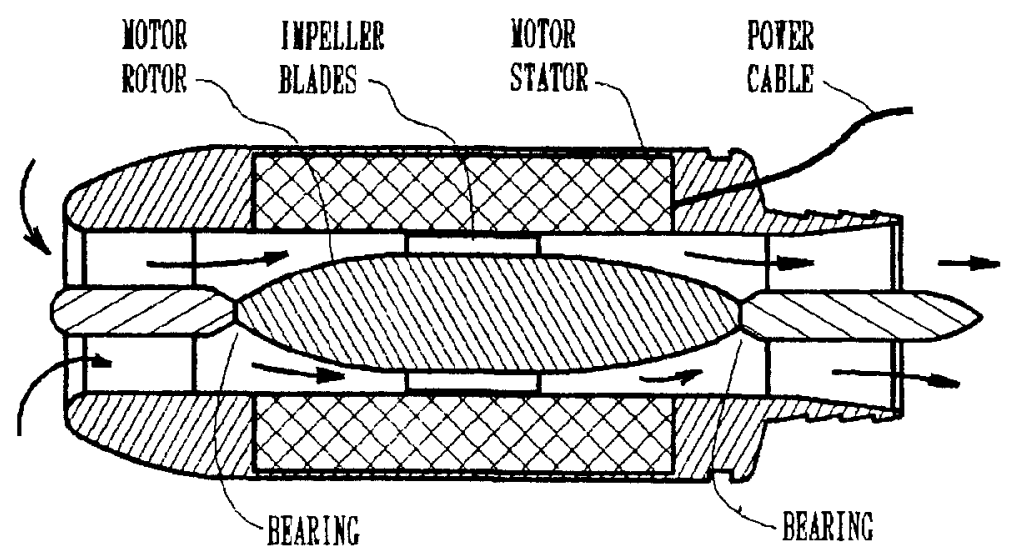

Fig. 1. Jarvik 2000 intraventricular axial flow assist device measures $1.8 \mathrm{~cm}$ in diameter by $5 \mathrm{~cm}$ in length and has displacement volume of $12 \mathrm{ml}$. Rotor is encased in titanium shell and is supported at each end by tiny blood-immersed ceramic bearing $1 \mathrm{~mm}$ in diameter.

date, however, experimental and clinical trials with axial flow pumps have demonstrated limited flows, hemolysis, platelet destruction, thrombus formation on impeller blades, device overheating, and limited duration of implantation. ${ }^{9-14}$

In response to the need for a ventricular assist device for smaller patients, we evaluated a novel axial flow intraventricular device, the Jarvik 2000 pump (Jarvik Research, Inc., New York, N.Y.), in an ovine model. Parameters addressed included safety, durability, hemolysis, thromboembolism, and power requirement.

\section{Material and methods}

Device. The Jarvik 2000 device is an intraventricular axial flow impeller pump that measures $1.8 \mathrm{~cm}$ in diameter by $5 \mathrm{~cm}$ in length, weighs $45 \mathrm{gm}$, and has a displacement volume of $12 \mathrm{ml}$ (Fig. 1). In vitro studies with this device have demonstrated flow rates from 2 to $7 \mathrm{~L} / \mathrm{min}$ in a water-glycerol test solution with viscosity similar to that of blood at $100 \mathrm{~mm}$ differential pressure. Normal operating speeds of 16,000 to $18,000 \mathrm{rpm}$ produce flow rates from 3 to $6 \mathrm{~L} / \mathrm{min}$ at $80 \mathrm{~mm} \mathrm{Hg}$ mean aortic pressure (Fig. 2). Power to the device is provided by a direct current power supply and regulated by a pulse width-modulated brushless direct current motor controller to set motor speed.

The device is placed into the apex of the left ventricle with outflow via a $12 \mathrm{~mm}$ Dacron graft to the descending thoracic aorta. Several stator blade and rotor configurations were tested in vitro and in vivo to minimize bearing wear, hemolysis, and power requirement.

Animals. In vivo studies with the Jarvik 2000 axial flow pump were conducted in mature sheep $(n=7)$ weighing $55.1 \pm 10.3 \mathrm{~kg}$. Surgical procedures and subsequent care of animals were done in compliance with the "Principles of Laboratory Animal Care" formulated by the National Society for Medical Research and the "Guide for the Care and Use of Laboratory Animals" prepared by the Na- tional Academy of Sciences and published by the National Institutes of Health (NIH Publication No. 85-23, revised 1985).

Operative procedure. Animals were sedated with ketamine hydrochloride $(10 \mathrm{mg} / \mathrm{kg}$ intramuscularly) and atropine sulfate $(0.4 \mathrm{mg} / \mathrm{kg}$ intramuscularly), anesthetized with inhalational isoflurane ( 0.5 minimal alveolar concentration) and the lungs ventilated through an endotracheal tube with an inspired oxygen fraction of $100 \%$. Animals were monitored continuously for arterial blood pressure and electrocardiogram.

Device placement was done initially with the use of cardiopulmonary bypass $(n=4)$; however, we found this to be unnecessary and the remaining animals were operated on without cardiopulmonary bypass $(n=3)$. After standard left thoracotomy, the animals were fully anticoagulated with heparin and the $12 \mathrm{~mm}$ Dacron outflow graft was preclotted with nonheparinized autologous blood. The descending thoracic aorta was partially occluded with a vascular clamp and the Dacron graft anastomosed to the aorta in an end-to-side manner.

The power cable was tunneled through the soft tissues and exteriorized on the left flank. In animals undergoing cardiopulmonary bypass, cannulas were placed in the external jugular vein and the ascending aorta or Dacron graft and bypass was initiated; in the remaining animals, lidocaine $100 \mathrm{mg}$ was injected into the pericardium to avoid ventricular arrhythmias during manipulation of the heart.

A silicone-reinforced Dacron velour sewing ring was attached to the apex of the left ventricle with braided polyester sutures supported with felt pledgets. An excisional apical left ventriculotomy through the sewing ring was created with a cylindrical coring knife through which the device was inserted. Bleeding from the apex was controlled by manual compression of the heart during device insertion. The device was secured to the sewing ring with umbilical tape purse-string ties. The outflow graft was vented to remove residual air and pumping was initiated. An ultrasonic flow probe (Transonic Systems, 


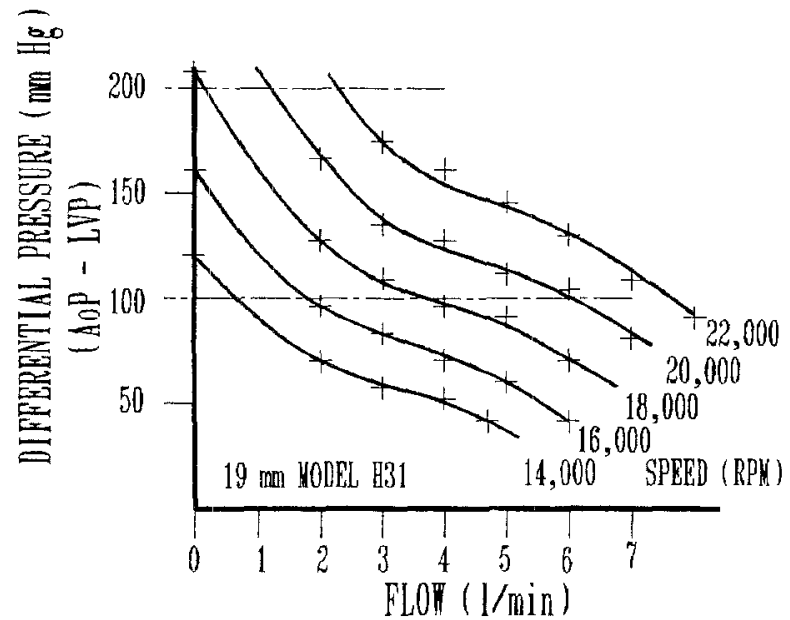

Fig. 2. In vitro studies with Jarvik 2000 intraventricular assist device have demonstrated flow rates from 2 to 7 $\mathrm{L} / \mathrm{min}$ in water "glycerol test solution with viscosity similar to that of blood. At normal operating speeds of 16,000 to $18,000 \mathrm{rpm}$, device produces flow rates from 3 to $6 \mathrm{~L} / \mathrm{min}$ at $80 \mathrm{~mm} \mathrm{Hg}$ mean aortic pressure. AoP, Aortic pressure; $L V P$, left ventricular pressure.

Inc., Ithaca, N.Y.; $n=3$ ) was placed around the graft, flow recorded, and the flow probe removed. A chest tube was placed for drainage, the incisions closed, and the animals returned to their cages.

Postoperative care and monitoring. Chest tubes were removed within 24 hours of operation. Heparin 1000 units per hour was started on the morning after the operation and continued for 3 days. Aspirin $325 \mathrm{mg}$ and warfarin sodium were given daily starting on the morning after the operation; warfarin sodium was dosed to maintain prothrombin times approximately 1.5 times the control value. Device motor speed, voltage, and current were monitored continuously. Serum samples were collected serially to determine blood cell counts, blood chemistry values, prothrombin times, and levels of plasma free hemoglobin.

\section{Results}

The animals survived from 3 to 123 days. The first two animals died as a result of technical complications on days 3 and 5, respectively. In the first sheep, the device was oriented with the inflow stator directed toward the interventricular septum. Septal compression led to infarction with subsequent right ventricular failure and death. The second animal died as a result of hemorrhage from the anastomosis of the Dacron graft and aorta.

The remaining animals survived $57 \pm 18$ days. Of these, the first animal was killed on postoperative day 42 when the power cable to the device broke at

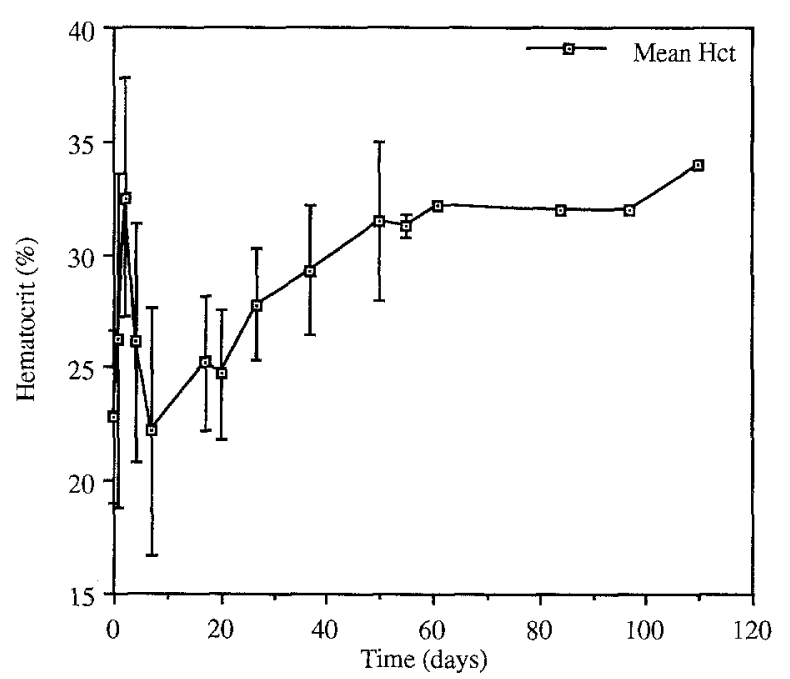

Fig. 3. Before placement of Jarvik 2000 intraventricular assist device, sheep were anemic with mean hematocrit (Hct) of $22.8 \% \pm 3.8 \%(n=7)$. During course of mechanical support, mean hematocrit values rose significantly to $30.5 \% \pm 3.4 \%(p=0.01)$.

the point of insertion into the pump, likely as a result of animal growth during the course of mechanical support and mechanical stress caused by movement. Similarly, the next animal was killed because of a broken power cable on postoperative day 19. This break occurred as a result of the cable having been brought out too low along the flank, which allowed the sheep to lie on the cord. A third animal was killed on postoperative day 58 when device function became erratic, which necessitated the use of increased power to maintain motor speed. Postmortem examination of this device demonstrated a small ring of thrombus at the inflow stator, presumably caused by turbulent flow at that point. The impeller blades were modified to further minimize flow turbulence and the next two animals did well until they were killed on days 123 and 42 because of broken power cables, again as a result of animal movement and growth during the course of mechanical support.

In all animals, a small ring of thrombus approximately $1 \mathrm{~mm}$ thick was present at the bearing that supported each end of the rotor. In the animal that survived 123 days, a thin capsule formed over the surface of the rotor hub but did not cover the impeller blades. None of the animals evidenced end-organ dysfunction clinically, and at autopsy no emboli were found in peripheral arteries. Two of the animals had mild, local skin infections at the power 


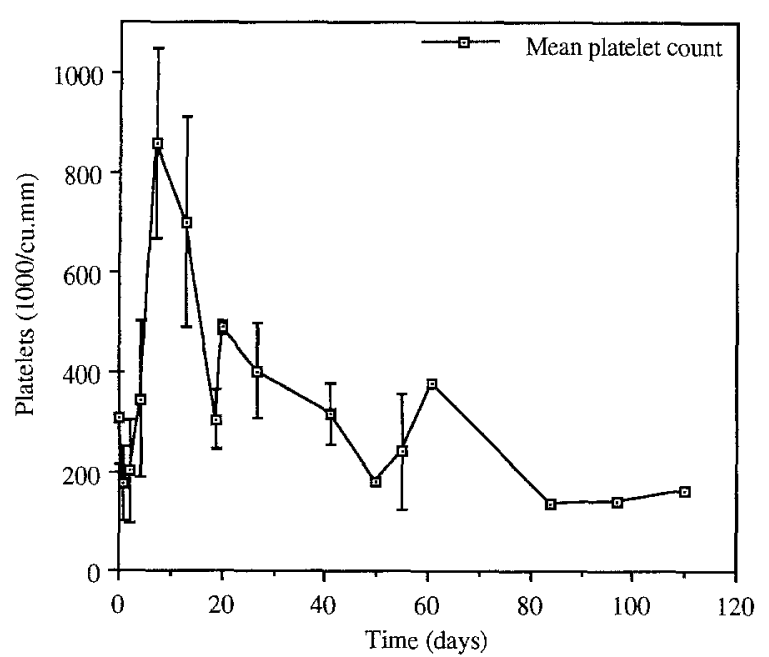

Fig. 4. Except for early perioperative thrombocytosis, platelet counts were normal in all sheep $(n=7)$. None of sheep had thrombocytopenia at any time while supported by Jarvik 2000 intraventricular assist device.

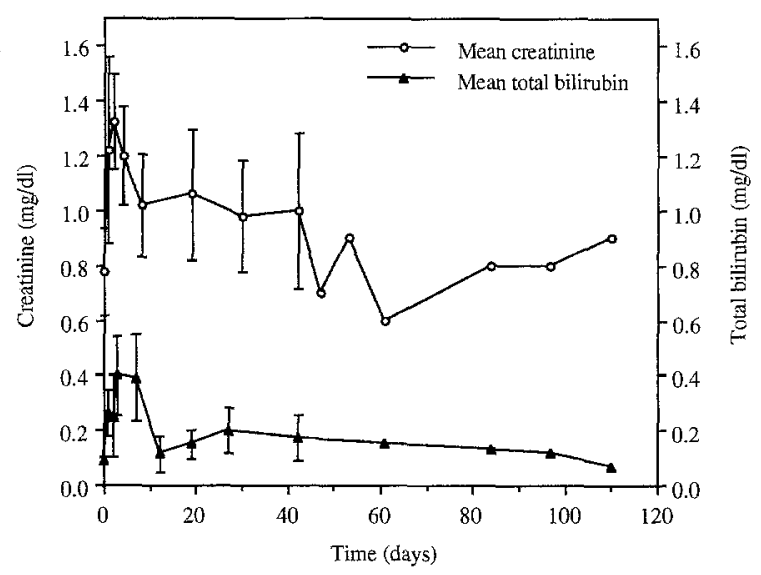

Fig. 5. Mean bilirubin and creatinine levels were within normal limits for all sheep $(n=7)$ during mechanical support.

cable egress site; however, these were easily treated with dressing changes.

The sheep were anemic before device implantation; however, during the time that the animals were assisted by the device, mean hematocrit values rose significantly from $22.8 \% \pm 3.8 \%$ before operation to $30.5 \% \pm 3.4 \%(p=0.01)$ before they were killed (Fig. 3). After an early thrombocytosis, platelet counts returned to normal (Fig. 4) and the animals were never thrombocytopenic. Bilirubin and creatinine levels remained within the control range (Fig. 5). Elevations higher than baseline levels of mean plasma free hemo-

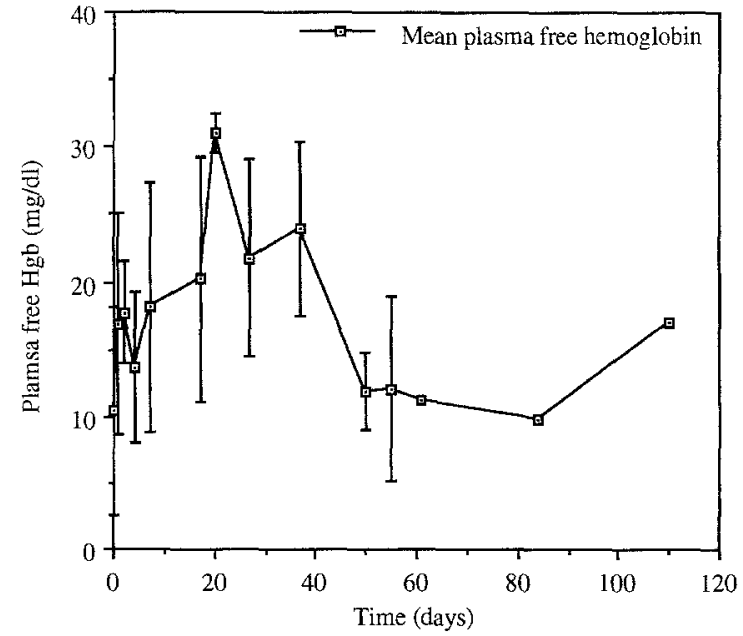

Fig. 6. Mean plasma free hemoglobin $(H g b)$ levels were insignificantly $(p=0.1)$ elevated in comparison with baseline levels for all sheep $(n=7)$ mechanically supported by Jarvik 2000 intraventricular assist device.

globin from $10.4 \pm 7.8 \mathrm{mg} / \mathrm{dl}$ to $17.1 \pm 7.4 \mathrm{mg} / \mathrm{dl}$ before the animals were killed were statistically insignificant ( $p=0.1$, Fig. 6 ) as were elevations in mean lactate dehydrogenase levels from $391.5 \pm 113.7$ units/L to $771.2 \pm 370.8$ units/L ( $p=0.1$, Fig. 7 ).

Flow through the Dacron graft, as measured intraoperatively with ultrasonic flow probes, validated the flow rates demonstrated in vitro (Fig. 8). In that preload to the device varied with native heart contraction, outflow from the device was pulsatile in nature. At higher speeds with the ventricle fully unloaded, however, flow from the device became nonpulsatile. When the device was momentarily turned off, flow through the Dacron graft was reversed. This functional "aortic insufficiency" was tolerated by the animals.

\section{Discussion}

The principle of operation of the Jarvik 2000 intraventricular assist device is extremely simple. The rotor of a brushless direct-current motor, a small high-strength magnet about $5 \mathrm{~mm}$ in diameter by $2 \mathrm{~cm}$ long, is encased in a titanium shell that supports the blades of a miniature axial flow pump. This constitutes the sole moving part of the device and is supported at each end by a tiny bloodimmersed ceramic bearing $1 \mathrm{~mm}$ in diameter. Because the bearings are so small, the surface rubbing speed even at $20,000 \mathrm{rpm}$ is very low and there is virtually no wear. The rotor is supported for rotation within the bore of the motor stator, which is also encased by titanium, and the forces to spin the rotor 


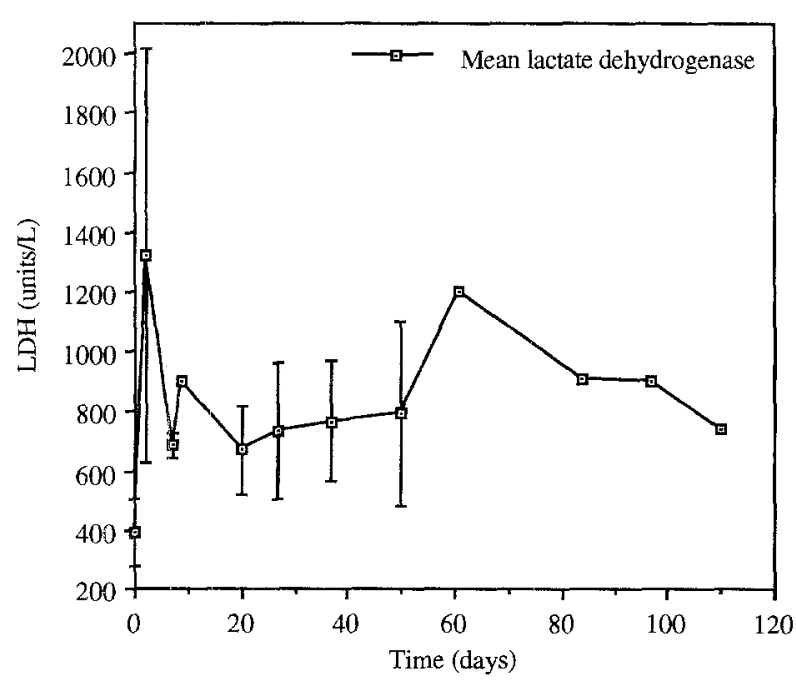

Fig. 7. Mean lactate dehydrogenase $(L D H)$ levels were insignificantly $(p=0.1)$ elevated in comparison with baseline levels in those sheep tested while mechanically supported by Jarvik 2000 intraventricular assist device.

are transmitted magnetically across the blood flowing through the bore of the motor. A small ring of thrombus is permitted to form immediately at the bearing sites, but this is in the form of a tiny torus that cannot break free and embolize because it is retained by the bearing shaft that passes through the center. A critical feature of the design is that a high-flow stream of blood passes across each bearing to prevent the thrombus from becoming much larger than the tiny gap it fills. One of the major objectives of this study was to establish that the flow in vivo was sufficient to properly wash the bearings.

In these animal experiments, we found the Jarvik 2000 intraventricular assist device to be entirely free from embolic complications, easy to manage in the long term, and able to deliver up to $5 \mathrm{~L}$ per minute of flow in seven sheep. Further, unlike currently available pusher-plate type LVADs, the Jarvik 2000 device is small enough to be implanted into patients with body surface areas less than $1.5 \mathrm{~m}^{2}$.

After initial experiments during which we learned how best to place this device in the heart, survival of the sheep was limited primarily by the animals' either chewing or breaking the power cables. Further device development with improved stress relief of the wires is under way. The device in the fifth animal likely failed because of a small ring of thrombus at the inflow stator, and after impeller blade modification no evidence of thrombus forma-

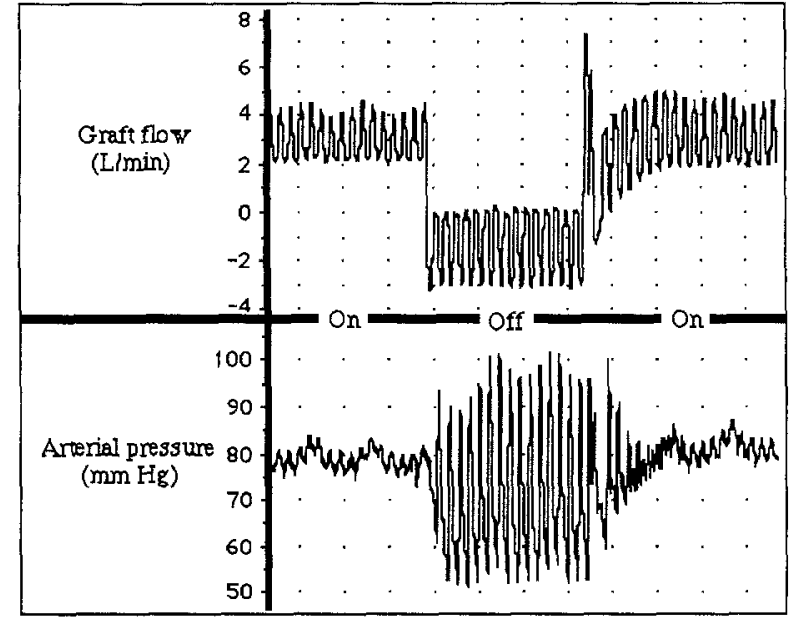

Fig. 8. Flow and pressure tracings recorded with Jarvik 2000 intraventricular assist device turned on and off showed that flow from device, in this model, was pulsatile in nature. This was probably function of varied preload to device resulting from contractility of normal native heart; at higher pump speeds, flow from device became nonpulsatile. When device was turned off, flow through Dacron graft was reversed as indicated by negative flow in flow tracing. This retrograde flow was tolerated by all animals.

tion was noted in subsequent animals except that of a small stable ring at the bearings.

The slight elevations of levels of plasma free hemoglobin and serum lactate dehydrogenase suggest that some hemolysis occurred. However, stable postoperative hematocrit values indicate that this small degree of hemolysis was not clinically significant.

None of the devices evidenced overheating or significant bearing wear. The minimal power requirements of the device ( $<10$ watts) would permit long-term use with battery power supply.

The arterial pressure tracings and flow probe recordings (Fig. 7) demonstrated that, in this model, flow from the device was pulsatile in nature. The pulse pressure recorded here was approximately 10 $\mathrm{mm} \mathrm{Hg}$ and although in the normal heart setting this is likely a result of residual myocardial function varying blood return to the device, at higher speeds with the ventricle completely unloaded we would expect a more continuous flow pattern. Nonetheless, pulsatile flow could be easily provided by cycling motor speed.

Flow through the graft with the device turned off was reversed, as indicated by the negative flow in the flow probe tracing (Fig. 7). Because there is no valve 
in the Dacron graft, when the ventricle ejects normally through the aortic valve, retrograde flow through the graft occurs. Although this functional "aortic insufficiency" was well tolerated by all of the animals, pump failure in the setting of heart failure would likely require immediate attention.

Whereas the goal of this initial investigation of the Jarvik 2000 intraventricular support device was to address safety, durability, hemolysis, thromboembolism, and power requirements, future studies to determine the utility of this novel axial flow intraventricular device should include investigations of longer-term animal survival and function in a smaller-animal, heart-failure model. We believe that the combination of ease of use, durability, and biocompatibility in smaller patients will make this pump a suitable assist device for patients unable to tolerate the currently available larger LVADs.

\section{REFERENCES}

1. Kaye MP. The Registry of the International Society for Heart and Lung Transplantation: tenth official report1993. J Heart Lung Transplant 1993;12:541-8.

2. Evans RW, Manninen DL, Garrison LP, Maier AM. Donor availability as the primary determinant of the future of heart transplantation. JAMA 1986;255:1892-8.

3. Schuler S, Parnt R, Warnecke H, et al. Extended donor criteria for heart transplantation. J Heart Transplant 1988;7:326-30.

4. Copeland JG, Emery RW, Levinson MM, Copeland J, McAleer MJ, Riley JE. The role of mechanical support and transplantation in treatment of patients with endstage cardiomyopathy. Circulation 1985;72(Suppl):II712.

5. Reemtsma K, Drusin R, Edie R, Bregman D, Dobelle W, Hardy M. Cardiac transplantation for patients requiring mechanical circulatory support. $\mathrm{N}$ Engl $\mathrm{J}$ Med 1978;298:670-1.
6. Miller CA, Pae WE, Pierce WS. Combined registry for the clinical use of mechanical ventricular assist pumps and the total artificial heart in conjunction with heart transplantation: fourth official report1989. J Heart Transplant 1990;9:453-8.

7. Frazier $\mathrm{OH}$, Branislav R. Ventricular assist devices. In: Cardiac Surgery: state of the art reviews. Philadelphia: Hanley \& Belfus, 1990;4:335-47.

8. $\mathrm{Oz} \mathrm{MC,} \mathrm{Rose} \mathrm{EA,} \mathrm{Levin} \mathrm{HR.} \mathrm{Selection} \mathrm{criteria} \mathrm{for}$ placement of left ventricular assist devices. Am Heart J 1995;129:173-7.

9. Arabia FA, Copeland JG, Larson DF, Smith RG, Cleavinger MR. Circulatory assist devices: applications for ventricular recovery or bridge to transplant. In: Gravlee GP, ed. Cardiopulmonary bypass: principles and practice. Baltimore: Williams and Wilkins, 1993:693-712.

10. Unger F, Genelin A, Hager J, et al. Functional heart replacement with nonpulsatile assist devices. In: Unger F, ed. Assisted circulation 2. Berlin: SpringerVerlag, 1984:163-74.

11. Frazier OH, Macris MP, Wampler RK, Duncan JM, Sweeney MS, Fuqua JM. Treatment of cardiac allograft failure by use of an intraaortic axial flow pump. J Heart Transplant 1990;9:408-14.

12. Butler KC, Maher TR, Borovetz HS, et al. Development of an axial flow blood pump LVAS. ASAIO J 1993;38:M296-300.

13. Wiebalck AC, Wouters PF, Waldenberger FR, et al. Left ventricular assist with an axial flow pump (Hemopump): clinical application. Ann Thorac Surg 1993;55:1141-6.

14. Golding LR, Jacobs G, Murikami T, et al. Chronic nonpulsatile blood flow in an alive awake animal: 34-day survival. Trans Am Soc Artif Intern Organs 1980;26:251-5. 